Herzschr Elektrophys 2012 $23: 259$

DOI 10.1007/s00399-012-0242-6

Online publiziert: 22. November 2012

c) Springer-Verlag Berlin Heidelberg 2012
Carsten W. Israel

Klinik für Innere Medizin - Kardiologie, Diabetologie \& Nephrologie,

Evangelisches Krankenhaus Bielefeld, Bielefeld, Deutschland

\section{Bildgebung in der Elektrophysiologie}

\section{Was können wir sehen?}

Wir sehen sie auf jedem Cover der großen internationalen elektrophysiologischen Zeitschriften die dreidimensionalen bunten Mapping-Bilder, mit und ohne hinterlegte MRT- oder CT-Bilder. Doch welchen Stellenwert haben die neuen bildgebenden Verfahren heute in der klinischen Routine? Brauchen wir sie? Die vorliegende Ausgabe von Herzschrittmachertherapie und Elektrophysiologie stellt den aktuellen wissenschaftlichen Stand hierzu dar.

Herr Gutleben fasst den Stellenwert der Echokardiographie in der invasiven Elektrophysiologie dar. Hier ist besonders die intrakardiale Echokardiographie (ICE) hervorzuheben. Mittels ICE können intrakardiale Strukturen, die entweder das Ziel oder ein Hindernis für die Katheterpositionierung darstellen können, unmittelbar sichtbar gemacht werden, von der Crista terminalis bei der rechtsatrialen Ablation über die Fossa ovalis (oder ein komplexes Tunnelsystem) bei der transseptalen Punktion bis zu Aortenklappe und linkskoronarem Hauptstamm bei der Ablation von Kammertachykardien. Vor allem aber können bei linksseitigen Ablationen „microbubbles“ und intraprozedurale Thromben am Ablationskatheter frühzeitig gesehen und kardioembolische Komplikationen so vermieden werden. Einen etablierten Stellenwert hat ICE auch bei der Pulmonalvenenisolation, bei der überprüft werden kann, ob die Ablation außerhalb der (teilweise anatomisch komplexen) Pulmonalvenenostien durchgeführt wird. Die Kombination von ICE und 3-D-Mappingverfahren kann eine hilfreiche Rekonstruktion der Anato- mie auch im Zusammenhang mit Voltage, Propagation und Ablationspunkten ermöglichen.

Moderne 3-D-Mappingsysteme wie Carto $^{\circledR}$ und $\mathrm{NavX}^{\circledR}$ erreichen in ihren aktuellen Versionen eine Auflösung und anatomische Genauigkeit von $<1 \mathrm{~mm}$. Herr Halbfass berichtet auch über neue spezielle Komponenten (z. B. „Merging“ zur Integration von MRT- oder CT-Bildern, Anpressdruckmessung) und die Kompatibilität mit Magnet- und Robotersystemen, die moderne Mappingsysteme bereichern.

Eine spannende Entwicklung betrifft die Nutzung der MRT für die Ablation: Mittels kontrastmittelverstärkter Spätaufnahmen kann mithilfe des MRT das Ausmaß myokardialer Fibrose z. B. vor Vorhofflimmerablation und die Transmuralität von Ablationspunkten nach Intervention beurteilen werden. Neue Techniken, Entwicklungen und Anwendungen der kardialen MRT in der Elektrophysiologie werden von Herrn Mahnkopf dargestellt.

Den Stellenwert der kardialen Computertomographie (CT) stellt Herr Schmidt in einem Übersichtsartikel dar, der insbesondere auf die Pulmonalvenenisolation fokussiert. Hier spielt das CT eine wichtige Rolle für die Planung und Durchführung der Prozedur. Mittels Integration anatomischer Bilddaten des CT in dreidimensionale Navigationssysteme kann sowohl die Fluoroskopiezeit reduziert als auch das Ablationsergebnis verbessert werden. Auch Komplikationen wie die Pulmonalvenenstenose und der Pulmonalvenenverschluss oder die Schädigungen von Ösophagus und N. phreni- cus können durch Kenntnis der Anatomie vermieden werden.

Eine neue Technik zur Bildgebung, MediGuide $^{\mathrm{TM}}$, ermöglicht nach Aufzeichnung von Fluoroskopieloops eine strahlungsfreie dreidimensionale Katheterdarstellung. Herr Sommer berichtet über Erfahrungen mit MediGuide ${ }^{\mathrm{TM}}$ bei der Ablation von Vorhofflattern, Vorhofflimmern und ventrikulären Tachykardien sowie bei der Implantation von linksventrikulären Elektroden bei CRT-Implantation. Unter anderem konnten die Durchleuchtungszeiten ohne Zunahme der Komplikationsraten deutlich reduziert werden.

Bildgebung spielt bei den komplexen Prozeduren der invasiven Elektrophysiologie eine immer größere Rolle. Der vorliegende Themenschwerpunkt gibt eine exzellente Übersicht über neue Methoden, deren praktische Anwendung und deren Potenzial. Herrn Brachmann, der als Gastherausgeber Themen und Autoren zusammengestellt hat, und den Autoren gilt ein herzlicher Dank für eine herausragende Leistung.

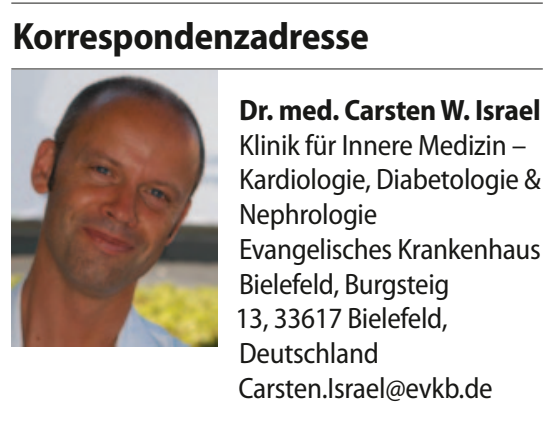

